\title{
Exploring the Economic Implications of a Group-Based Lifestyle Intervention for Middle-Aged Adults with Chronic Schizophrenia and Co-Morbid Type 2 Diabetes
}

\section{A-La Park*}

London School of Economics and Political Science, Personal and Social Services Research Unit, UK

\begin{abstract}
Objective: People with schizophrenia are at elevated risk of having type 2 diabetes, compared to the general population. Although there is a growing body of literature on the effectiveness of behavioural interventions to improve physical activity levels and healthy eating habits, there are very few economic evaluations conducted for people with severe mental illness and physical health problems. The aim of this study is to explore the cost-effectiveness of group-based lifestyle interventions for middle-aged adults with enduring long-term schizophrenia and co-morbid type 2 diabetes.
\end{abstract}

Methods: Deterministic decision analytical modelling techniques are applied to explore cost-effectiveness based on the findings from a systematic review on this population. The intervention refers to a group-based lifestyle intervention, which is a combination of exercise, diet and diabetes education over 6 months. The intervention costs are estimated to reflect the UK local context.

Results: From the UK National Health Service perspective, the group-based lifestyle intervention is potentially cost-effective, relative to usual care. It costs $£ 80$ to achieve an additional unit of Body Mass Index (BMI) lost and the incremental cost-effectiveness ratio is just below $£ 700$ to gain an extra Quality Adjusted Life Year, which lies well within the conventionally acceptable threshold values in the UK.

Conclusions: Group based life style intervention for this population is effective and can be cost-effective in reducing $\mathrm{BMI}$ and improving diabetes knowledge, at least in the short-term. More co-ordinated policies among relevant sectors are required to facilitate behavioural change and better maintain an improved lifestyle. An integrated approach is needed to make this more sustainable in the long-term.

Keywords: Economics; Exercise; Lifestyle; Group-based; Middleaged adults; Nutrition; Schizophrenia; Severe mental illness; Type 2 diabetes

\section{Introduction}

People with schizophrenia are at elevated risk of developing type 2 diabetes. The prevalence of type 2 diabetes is more than two times greater in this population compared to the general population [1]. Enduring mental illness and chronic physical morbid conditions contribute to this double burden, not only to individuals but to society as a whole. Patients themselves and families and carers also experience lower quality of life and productivity losses. The total societal costs related to diabetes in the UK currently are $£ 23.7$ billion and are estimated to rise to $£ 39.8$ billion by 2035 -2036.Around $10 \%$ of the National Health Service (NHS) budget is spent on diabetes [2]. In particular, older adults aged 40 and above are at increased risk of diabetes partly because of their long-term use of antipsychotics and advancing age [3].

Antipsychotic-induced weight gain may result in voluntary unplanned withdrawal of drug regimens, which in turn lead to higher relapse rates and incur more costs to health and social care services. Weight gain can negatively affect various aspects of individuals' lives including physical health per se, as well as their body image, perception, confidence and social life. Increased metabolic risk factors are associated with other physical disorders such as cardiovascular diseases and reduced life expectancy of up to 10 years in people with type 2 diabetes [4] and up to 15-20 years due to schizophrenia [5]. As a result of poor physical health, they are less likely to be employed. They are more dependent on social security benefits.
Lifestyle interventions are effective for people with type 2 diabetes in the general population. Lifestyle interventions to promote physical activity and healthy eating habits though motivational interviewing techniques have been shown to be beneficial for people with mental health problems and comorbid somatic disorders [6]. Moreover, there is a growing number of studies on the clinical effectiveness of exercise, diet, psycho-social education, and counselling approaches or combined interventions of more than two of these components for people with severe mental illness. Recent systematic reviews suggest positive impacts on weight loss, BMI, glucose tolerance etc. $[7,8]$.

Exercise alone is less effective than combined lifestyle interventions [9], while nutrition education alone does not make a significant difference in outcomes [10]. So combination therapy is more effective in achieving the desired outcomes. Multimodal approaches/multifactorial interventions are needed to meet the needs of people with complex needs.

*Corresponding author: A-La Park, Personal and Social Services Research Unit LSE Health and Social Care, London School of Economics and Political Science, Houghton Street, London, WC2A 2AE, UK, Tel: ++44(0)20-7849-4665; Fax: +44(0)20-7955-6131; E-mail: A.Park@lse.ac.uk

Received April 08, 2014; Accepted April 30, 2014; Published May 05, 2014

Citation: Park AL (2014) Exploring the Economic Implications of a Group-Based Lifestyle Intervention for Middle-Aged Adults with Chronic Schizophrenia and CoMorbid Type 2 Diabetes. J Diabetes Metab 5: 366 doi:10.4172/2155-6156.1000366

Copyright: (c) 2014 Park AL. This is an open-access article distributed under the terms of the Creative Commons Attribution License, which permits unrestricted use, distribution, and reproduction in any medium, provided the original author and source are credited. 
In the UK, the physical health section of the most recent National Institute for Health and Care Excellence guideline [9] for people with psychosis or schizophrenia recommends that, those especially taking antipsychotics, should be offered a combined healthy eating and physical activity programme by their mental healthcare provider and regular monitoring of metabolic indicators in a primary care setting. But there is very limited economic evidence to justify the programme. In particular, it does not mention any specific strategy for particular sub-population groups including older adults who have both chronic schizophrenia and type 2 diabetes. The aim of this study is to explore the cost-effectiveness of group-based life style interventions for middleaged adults with chronic schizophrenia and co-morbid type 2 diabetes, using a decision modelling approach. Such a modelling approach could then be further utilised and adapted for different contexts and clinical settings.

\section{Methods}

Decision analytical models were constructed to explore costeffectiveness by comparing the costs and outcomes of group-based lifestyle intervention with usual care, using TreeAge Pro 2014 [11]. This kind of modelling approach can be helpful when there is limited availability of data from empirical studies such as randomised clinical trials or quasi-experimental settings. Although trial-based approaches can have better internal validity, they are more likely to be resource intensive and tend to take a longer time to identify clinically meaningful results. On the other hand, decision analytical models can be generated within a relatively shorter time period, compared to trial-based approaches. It is very useful when decision makers such as policymakers, commissioners and funders -need to know the most plausible outcomes given a relatively short time frame before making investment decisions on new interventions/programmes in the absence and/or a lack of long term empirical data . One of the advantages of the modelling approach is better external validity as the model can be adapted from one country setting to another and/or from inpatient care to outpatient care settings.

\section{Intervention}

The intervention was based on a diabetes awareness and rehabilitation training programme, consisting of twelve 90-minute sessions of a manualised healthy lifestyle programme over a six-month period. Modules of the programme included psycho-social education on diabetes, healthy eating habits and physical activity [12,13]. Selfmonitoring one important component of the programme which provided opportunities to learn about appropriate portion sizes for various food groups, reading food product labels, replacing sugary snacks with more fibre rich foods, as well as how to keep track of physical activity levels on a daily basis. For example, study participants could self-record their level of exercise using pedometers, which could facilitate their empowerment. Raffle tickets were offered to the participants as rewards to encourage their constant attendance. This intervention was compared with usual care. This refers to two General Practitioner (GP) visits with $20 \%$ of patients referred to dieticians. This is currently recommended as routine care in NICE guidance in the UK $[9,14]$. It does not include any specific component particularly targeted at people with both schizophrenia and diabetes.

\section{Effectiveness data}

Data regarding people with schizophrenia and type 2 diabetes were obtained from two studies in a systematic review $[12,13,15]$. Six months after the end of the studies, Body Mass Index (BMI) of service users receiving the programme reduced on average from $33.6 \mathrm{~kg} / \mathrm{m}^{2}$ to $32.9 \mathrm{~kg} / \mathrm{m}^{2}$, while BMI of those receiving usual care was increased on average from $33.6 \mathrm{~kg} / \mathrm{m}^{2}$ to $33.9 \mathrm{~kg} / \mathrm{m}^{2}(\mathrm{p}<0.001)$. Although the level of knowledge on diabetes in the intervention group slightly decreased at 12 months from 0.7 to 0.6 , it was still higher than the value of 0.5 at the baseline, whereas there was nochange in the scores for the control group $(\mathrm{p}<0.001)$. Therefore, this indicates that sustainable skills were gained via the programme.

\section{Costs}

Intervention costs were estimated assuming 4 concurrent groups were run by one specialist educator with 6 to 8 participants per group per session. Four sessions for each of the three different topics on diabetes education, nutrition, and lifestyle exercise were run over 24 weeks (i.e. in total 12 sessions). Specialist mental health professional's time costs of $£ 107$ were multiplied by 12 sessions to estimate the costs per group. Then these were multiplied by four concurrent groups. The total costs of $£ 5136$ were divided by the total maximum number of 32 participants in the experimental group. An extra $£ 12$ was added to individual costs as $\$ 20$ was offered to all participants for the follow-up visit. Although other costs associated with running the sessions such as hourly rent for premises or use of pedometers, and overheads for printing hand-outs could be included, they are a relatively minor part of the total costs, as the total costs are mainly driven by human resource costs to implement the sessions. Cost associated with diabetes were considered; both costs of diabetes with complications or without complications, based on the data in the UK Prospective Diabetes Study and NICE guidelines $[9,16]$. Unit costs were obtained from the Personal Social Services Research Unit health and social care costs [17]. All costs were adjusted to 2012-13 prices sterling pounds using the International Monetary Fund inflator. Discounting was not applicable as the time horizon was not beyond one year. Variables used in the model are presented in Table 1.

\section{Cost-Effectiveness Analysis (CEA) vs. Cost-Utility Analysis (CUA)}

In this study, the results are presented as in the form of costeffectiveness analysis and cost-utility analysis. To briefly explain the terms, cost-effectiveness analysis reports study results as cost per change in some form of clinical unit. In other words, it is related to the question of whether it is worth paying for an additional unit of benefit pernatural unit of outcome measure gained, in this case in terms of BMI loss achieved in this study. On the other hand, CUA presents the results as cost per Quality Adjusted Life Year (QALY) gained. That is to say; the cost of gaining one additional year of full quality life. -Incremental costeffectiveness ratios (ICERs) can act as summaries of the study findings. They can be expressed as the difference in costs against the difference in effects between the experimental and control groups (GL vs. UC).

\begin{tabular}{|l|c|c|}
\hline Variables & Values & Source \\
\hline Cost of Group-based lifestyle intervention (GL) & $£ 173$ & 13 \\
\hline Cost of usual care (UC) & $£ 147$ & 14 \\
\hline Cost of diabetes without complications & $£ 115$ & 9,18 \\
\hline Cost of diabetes with complications & $£ 2120$ & 9,18 \\
\hline Probability of uptake in GL & 0.53 & 12 \\
\hline Probability of BMI loss achieved in GL & 0.40 & 13 \\
\hline Probability of BMI loss achieved in UC & 0.15 & 13 \\
\hline Utility scores of BMI loss achieved & 0.80 & $9,18,19$ \\
\hline Utility scores of no BMI loss achieved & 0.67 & 9,19 \\
\hline
\end{tabular}

Table 1: Parameters used in the model (£2013 prices). 
Citation: Park AL (2014) Exploring the Economic Implications of a Group-Based Lifestyle Intervention for Middle-Aged Adults with Chronic Schizophrenia and Co-Morbid Type 2 Diabetes. J Diabetes Metab 5: 366 doi:10.4172/2155-6156.1000366

Page 3 of 7

\section{Decision tree model structure}

A decision model is constructed to estimate the cost-effectiveness of the group-based life style intervention, relative to that of usual care for people aged 40 and over with chronic schizophrenia (mean duration of 17 years) and type 2 diabetes (mean duration of 8 years). They are allocated into two groups: one including group-based lifestyle intervention (GL) and the other usual care (UC). Participants in each group can choose to uptake the services or not and then BMI loss may or may not be achieved through intervention. In each care pathway, people incur different costs and have corresponding values of effectiveness over 6 months. The tree is 'rolled back' to reveal the expected values for cost-effectiveness of the group-based lifestyle intervention, relative to usual care. The model was constructed and analyses were performed using the Data pro 2014 version [11]. The structure of the model is illustrated in Figure 1.

\section{Sensitivity analyses}

Uncertainty around the parameters used in the model can be explored using deterministic analyses such as one (two)-way sensitivity analyses and/or more dynamic sensitivity analyses using Monte-Carlo simulation. Static analyses use a range of fixed values for variables of interests. One-way sensitivity analysis can be useful to see what impact one variable has on the results within the fixed range of the specific parameter. In two-way sensitivity analysis, the values for two variables selected from the model can be changed at the same time by defining the possible ranges. The region graph in the two-way sensitivity analysis can identify any possible changes in the optimal strategy, which may change the plausible conclusion of the findings at base case analysis when the values for the two parameters of interest can be varied. On the other hand, dynamic probabilistic analyses use distributions of parameters of interests, which can be varied by taking into account the values for mean and standard deviations to explore uncertainty around the values used in the model.

\section{Cost-effectiveness plane}

In the cost-effectiveness plane, which will be used to report the results later, there are four quadrants including North West (NW), North East (NE), South West (SW) and South East (SE). The dots in the $\mathrm{NE}$ area indicate that a new intervention or programme is more costly and more effective, relative to the existing usual care or comparator(s) chosen for the analysis. Those in NW mean that a new intervention can be more costly and less effective, in which case economists would say

\begin{tabular}{|c|c|c|c|c|c|}
\hline \multicolumn{6}{|l|}{ Cost BMI loss achieved } \\
\hline Strategy & cost & effect & incrCost & incrEff & ICER \\
\hline Usual Care & 1195.21 & 0.08 & 0.00 & 0.00 & 0.00 \\
\hline Group-based lifestyle intervention & 1205.97 & 0.21 & 10.76 & 0.13 & 81.23 \\
\hline \multicolumn{6}{|l|}{ Cost per QALY gained } \\
\hline Strategy & cost & effect & incrCost & incrEff & ICER \\
\hline Usual Care & 1195.21 & 0.68 & 0.00 & 0.00 & 0.00 \\
\hline Group-based lifestyle intervention & 1205.97 & 0.70 & 10.76 & 0.12 & 676.89 \\
\hline
\end{tabular}

Table 2: Results of cost-effectiveness analysis and cost-utility analysis at base case (£2013 prices)

that the new intervention is "dominated" by usual care. In other words, usual care should be a preferred choice. Dots in the SW quadrant indicate that the new intervention is less costly and less effective. Those in the SE area mean that the new intervention can be more effective and less costly, which is a "dominant" option. Especially when dots are in the NE and SW quadrants, they are more likely to involve judgements based on how much money that a society is willing to pay for the particular programme. In England, the threshold values are approximately between $£ 20,000$ and $£ 30,000$ per QALY gained, as recommended by NICE. But the ceiling values vary from country to country. For example, in the USA, the preferred value is $\$ 50,000$ per QALY gained [20] and in most of the European countries, the acceptable threshold values are roughly $€ 30,000 / \mathrm{Q} A L Y$. It should be born in mind that these conventionally accepted threshold values are not fixed in stone. Of course, they are flexible in a sense that there is room for reflecting local priorities, depending on epidemiological factors and how the health and social care systems are financed in different countries.

\section{Results}

\section{Basecase}

In the base case scenario, the difference in costs between GL and UC can be divided by the difference in effectiveness between the two groups to get the Incremental Cost-effectiveness Ratio (ICER). The results of the cost-effectiveness analyses can be positive as the ICER is $£ 81.23$ to achieve an extra unit of BMI loss. This will depend on how much a decision-maker is willing and able to pay for an improvement in this outcome as shown in Table 2.

ICER $=\frac{\text { much a decision-maker is willing and able to pay for an imp }}{\text { Effect of intervention }- \text { Effect of control }}$

Similarly, in Table 2 the results of the cost-utility analysis can be

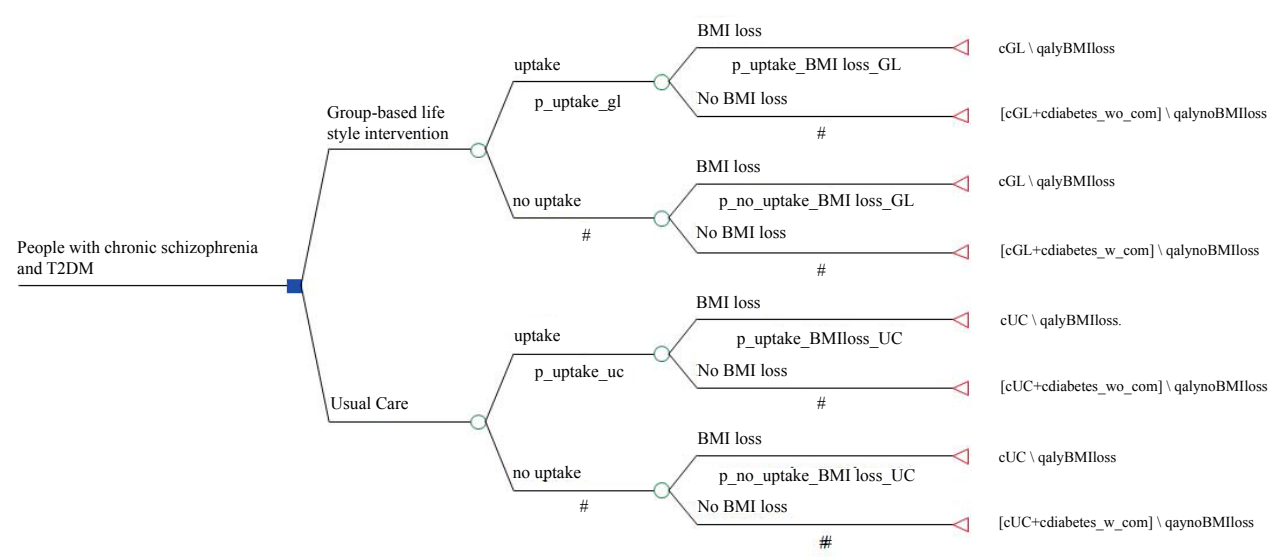

Figure 1: Decision analytical model for group-based lifestyle intervention vs. usual care. 
interpreted as GL being a very cost-effective option as the ICER is just below $£ 700$, which lies well below the conventionally accepted threshold values of $£ 20,000$ to $£ 30,000$ per QALY in the UK.

\section{Sensitivity analyses}

Following the famous quote by George Box "Essentially, all models are wrong, but some are useful" [21]. Economic models are a simplification of reality based on what is available for the modelling exercise. Therefore it is very important to explore uncertainty around the parameters used in the model by varying the assumptions about the variables of interests to help us say how confident we are about the results.

First, the costs of GL and UC are varied from the best scenario to the least optimistic scenario by assuming different unit costs applied to different health professionals' time costs and also different ratios in a group session. When the 12 -session programme with 4 groups is run by GP nurses in a primary care setting with an hourly rate of $£ 34$ ( $£ 51$ for 90 minutes), the intervention will cost $£ 76.50$ per participant. Similarly, if the programme is delivered by psychiatric nurses with an hourly rate of $£ 35$ ( $£ 52.50$ per 90 minutes), the cost will be $£ 78.75$ per person in the intervention arm.

The minimum costs can be applied when 4 GP nurses run the sessions at $£ 76.50$ per participant, assuming $100 \%$ attendance rates for a group of 8 people. The maximum costs of $£ 1,152$ per person can be applied when interventions are delivered by non-specialists for behavioural activation, assuming $£ 192$ per hour face-to-face contact (£288 per 90 minutes) with $50 \%$ of attendance rates for 6 people per group.

In a one-way sensitivity analysis, holding the costs of usual care constant and varying the costs of intervention from $£ 75.50$ to $£ 1152$, when primary care nurses or psychiatric nurses deliver the programmes, GL is a dominant strategy as GL is less expensive and more effective. At the maximum cost of $£ 1,152$, assuming 4 non-specialists deliver the behavioural activation programmes on a face- to- face contact basis

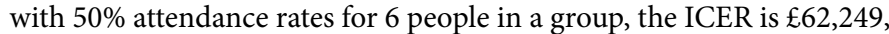
which is no longer cost-effective, given the acceptable ICER of $£ 30,000$ per QALY in England. However, when the cost of GL is $£ 399.13$, the ICER is $£ 14,900$ /QALY and at the cost of $£ 614.25$ in GL per person, the ICER is $£ 28,428$, which is just below $£ 30,000$ /QALY.

In a one-way sensitivity analysis, holding the intervention costs fixed, the cost of usual care can be varied from $£ 69$ to $£ 658$. The minimum cost can be applied when GP costs are assumed to be $£ 27$ per telephone consultation lasting 7.1 minutes, combined with referrals of $20 \%$ these patients to dieticians. On the other hand, the maximum cost can be up to $£ 658$ when GP costs are assumed to be $£ 292$ per hour of patient contact, including out of surgery costs such as clinics and home visits plus the costs for referring $100 \%$ of patients to dieticians.

The ICERs range from $£ 5,583$ per QALY at the usual care cost of

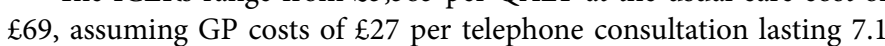
minutes, combined with $20 \%$ referrals to dieticians to usual care being dominated at $£ 658$ when GPs have visits to home or clinic plus $100 \%$ referral to dieticians. When the cost for usual care becomes $£ 187$, UC starts to become less effective and more costly, relative to GL.

Threshold analysis may be useful in the absence of empirical evidence to know when the results can be reversed from acceptable to less acceptable and vice versa. When an intervention or programme is under consideration for possible roll-out on a bigger scale than a small experimental setting to maximise the benefits from the

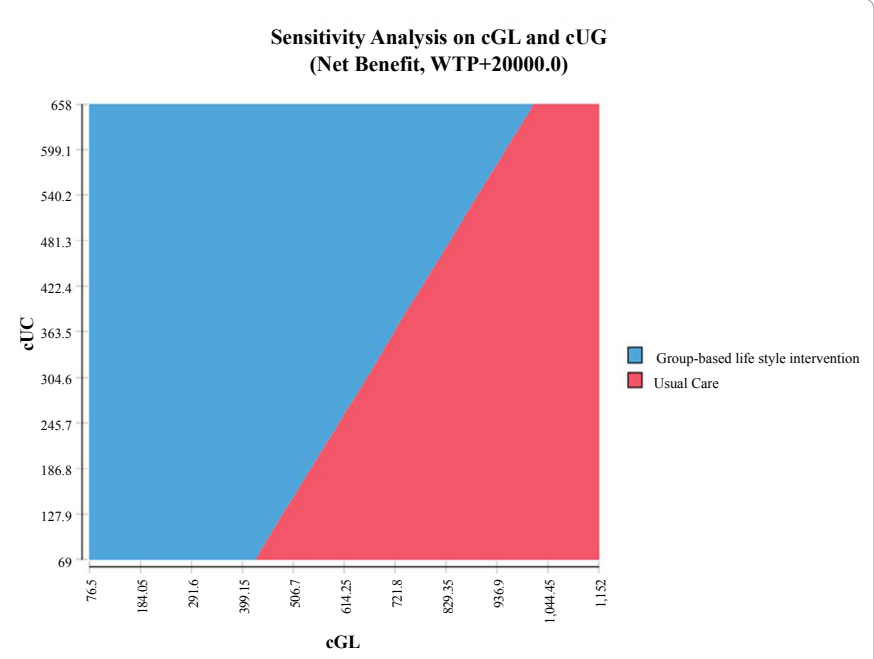

Figure 2: Two-way sensitivity analysis for costs of group-based lifestyle intervention and usual care.

intervention, it is important to know the optimal rate of uptake. Even if there is an intervention, which is proved to be clinically effective in a randomised controlled trial, if it is not used to the maximum capacity, the resource and costs for the implementation may not be efficiently used. Therefore the higher the uptake rate, the more costeffective the intervention is.

In two-way sensitivity analysis, two parameters can be selected such as cost of GL and cost of UC. When the values for these two variables are changed in a simultaneous fashion within the defined ranges, the region as shown in Figure 2 can efficiently identify any possible changes in the optimal strategy that should be considered for recommendation as the values of the two parameters change. Thresholds are simply the diagonal line between two areas.

Probabilistic sensitivity analysis can explore the uncertainty around the parameters of interests by using the Monte-Carlo Simulation technique. In this study, the main difference between deterministic and probabilistic sensitivity analyses is assessed using distributions of parameters such as the cost of GL, UC, and the probability of uptake in the GL group and quality of life score associated with BMI loss achieved, instead of using the actual fixed values. Figure 3 shows the scatter plot of the simulated pairs of costs and effects after 1000 iterations.

The cost-effectiveness plane as shown in Figure 3 illustrates the relative position of the new intervention, compared with existing usual care (from the origin). The graphical presentation of the simulated pairs indicates that most of the spots can be seen in the South area. While the dots in the SW quadrant indicate GL can be less costly and less effective, dots in the SE quadrant mean potentially that GL can be more effective and less expensive, which is a dominant strategy for the particular target population that we are looking at in this study.

At a willingness-to- pay of $£ 20,000$ per QALY gained, there is a $52.8 \%$ likelihood of the intervention being cost-effective. Therefore a decision on whether a particular programme should be recommended for consideration depends on judgement in the local contextto reflects the needs and/ or priorities for the local sub-population groups. Costeffectiveness analyses results can be used one as one of many inputs to feed into the complex decision making processes involving multiple stakeholders. 


\section{Incremental Cost-Effectiveness, Group-based life style intervention v. Usual Care}

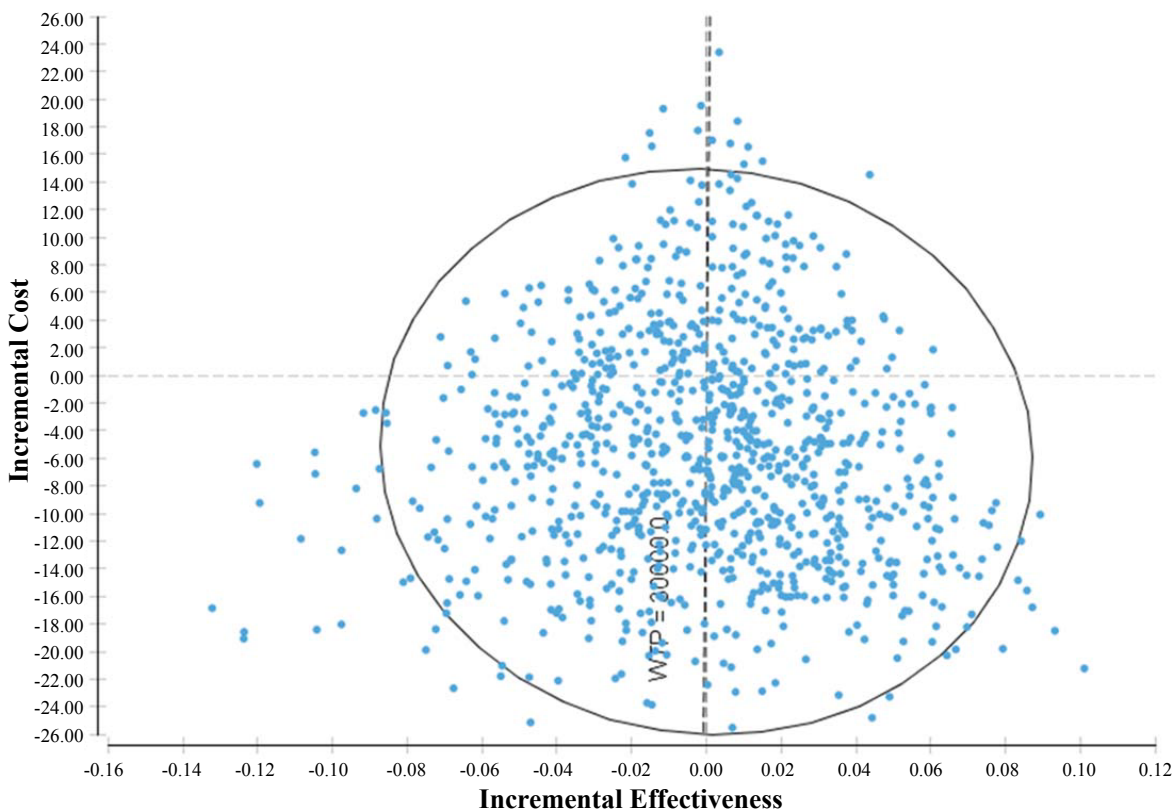

Figure 3: Scatter plot of Monte Carlo simulation results on the cost-effectiveness plane for group-based lifestyle intervention vs. usual care model.

\section{Discussion}

The results of decision tree models can be used to feed into the economic decision making process as one of the inputs when decision-makers need to make a strategic decision on whether a new intervention, relative to alternative existing care option(s), should be funded within a relatively short period of time given a lack of empirical trial-based data. The modelling process is a simplified version of reality as it is not possible to incorporate all the possible variables and it is often not possible to know for certain what it would happen in the absence of particular data on possible scenarios. So one of the key advantages of using decision-analytical modelling is that models can explore what would happen under different assumptions within a relatively short period of time in a less resource intensive manner. But they are greatly influenced by the assumptions behind the values of particular parameters. It can be a very useful tool to -show the likely scenarios when decision makers such as commissioners, funders and policy-makers look for help to make strategic investment decisions.

From the NHS perspective, in the base case analysis, a groupbased lifestyle intervention (GL) for middle-aged adults with chronic schizophrenia and type 2 diabetes is more effective at modest cost in achieving an additional case of BMI loss and gaining an extra QALY, compared with usual care. The incremental cost per QALY gained

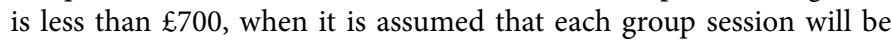
attended by eight people who will complete a 12 -session course of the therapeutic intervention. However, even with a lower rate of attendance up to $25 \%$ in a group for 8 people, the intervention will remain costeffective at a willingness-to-pay of $£ 30,000$ per QALY.

Depending on the types of health care professionals, the intervention costs can be more costly or cost-effective. In the sensitivity analysis, it appears that the intervention costs can be minimised when the manualised lifestyle programme is run by nurses in GP surgeries or in mental health clinics. When primary care nurses or psychiatric nurses deliver the programmes, GL is a dominant strategy as GL is less expensive and more effective. When varying the costs of the intervention, the same effectiveness size is assumed as the differences in the effectiveness are not well studied so far when the interventions are delivered by different health professionals.

At the policy level, it is important to promote sustainable lifestyle changes over the long-term by reducing barriers to uptake of those interventions in term of addressing affordability issues and access to necessary care services. The positive lifestyle changes achieved through lifestyle programmes can be further enhanced when governments at the policy level can provide more strategic support in terms of offering food subsidies for healthy fruit and vegetables, enforcing tax policies on foods with excessive amounts of sugar and salt, legislating for healthy foods and providing safe places to exercise more in the community for people with complex needs such as those on low income or with competing priorities in daily life.

The Canadian Population Health Survey (NPHS) from 1994/95 to 2006/07 highlighted the social determinants of health including inadequate income, insecurity associated with food and housing conditions as being among the most significant contributors to diabetes by showing the strong links between deprivation and diabetes. People living in poverty had a $41 \%$ higher likelihood of developing type 2 diabetes [22,23]. Similarly, Diabetes UK [3] also reported that women in England in the most deprived areasare four times more likely to have diabetes than those who live in the most affluent area.

A review by de Hert et al. [1] reported health inequalities in terms of access to information and services for diabetes among people with severe mental illness. People with schizophrenia are less likely to receive diabetes education within the past 6 months [24]. As a result, they tend to end up incurring more costs related to hospitalisation to treat diabetes complications. To narrow the gap in the services for this population, government should take a more active role in implementing educational programmes for related health professionals 
Citation: Park AL (2014) Exploring the Economic Implications of a Group-Based Lifestyle Intervention for Middle-Aged Adults with Chronic Schizophrenia and Co-Morbid Type 2 Diabetes. J Diabetes Metab 5: 366 doi:10.4172/2155-6156.1000366

Page 6 of 7

such as GPs, nurses, and cardiologists, diabetologists, psychiatrists and other specialists at the system level.

\section{Conclusion}

The mechanisms by which type 2 diabetes develops are interplay of genetic, biological, physical, social environmental factors. Although different antipsychotic drugs may have different levels of impact on metabolic risk factors, weight gain and diabetes, the mechanism for antipsychotic-induced diabetes is not clear [25]. However, lifestyle interventions were found to be effective, regardless of the type of antipsychotic drug and diabetes treatment regimens $[12,13]$.

A group-based lifestyle intervention is a promising option for middle-aged adults with chronic schizophrenia and comorbid type 2 diabetes, considering BMI loss achieved and QALYs gained. Refresher sessions may help prolong the beneficial effects accrued beyond 6 months, but little is known empirically about the long-term benefits or about the level of continued participation in these sessions. Studies are also currently focusing on how to normalise changes in lifestyles so as to make it easier to maintain change in the long term, drawing on work done by behavioural psychologists [26]. However, in the UK, a study by Holt et al. [27] found that a behavioural treatment programme for overweight and obese people with severe mental illness found significant progressive improvements in average weight and BMI over a 8- year follow-up period without any suggestion of a plateau $(\mathrm{p}<0.0001)$. This indicates promise for people with severe mental illness and diabetes. More empirical studies are also needed to confirm the long term costs and benefits of the impact of these physical health interventions on outcomes and costs over a longer time period. The lack of studies so far is not surprising - the same finding was reported in a review of the costs and benefits of interventions tackling co-morbid diabetes and depression [28]

For policy and practice, it would be more cost-effective if the intervention can be run at larger clinics than individual GP practices to reach the critical mass to justify the efficient use of resources for the likely number of people with both schizophrenia and co-morbid diabetes. Clearly, there are additional intangible benefits from a lifestyle intervention when it is implemented through a group rather than on an individual basis in terms of enhancing social capital and social integration to the communities, to which study participants belong. Group settings can have positive impacts on motivation levels and improve attendance rates. A group session can reduce staffing time, and allow service users to discuss their challenges and celebrate their accomplishments together, encourage behavioural changes and therefore promote peer-support [27]. But these intangible benefits are methodologically challenging to capture in monetary terms. If incorporated, it makes intervention even more cost-effective.

It is also important to take more personalised approaches to promoting self-efficacy and service user empowerment alongside broad strategic guidance. There are arguments for adopting patient-tailored rather than guidance-led treatment strategies [29]. Reflecting service users' choices by taking into account their personal circumstances and preferences in the future programmes may lead to more feasible and persistent behavioural changes. For instance, involving patients in the stage for the design of the programme can be one way to incorporate their preferences such as various types of exercise (indoor vs. outdoor sports, meditation/energy based slow movements vs. dynamic aerobic activities), monitoring by offline contacts vs. communications via mobile or internet in virtual community etc. This kind of bottom-up approach can allow more flexibility to refine the optimal policy for specific sub-populations and increase motivation levels of patients as an active service user.

Group based life style intervention for this population is effective and can be cost-effective in reducing BMI and improving diabetes knowledge at least in the short-term. More co-ordinated policies among relevant sectors beyond health care are required to facilitate behavioural changes and better maintain the improved lifestyle. More studies are needed to confirm cost-effectiveness in diverse ethnic groups, different age bands and with a longer term follow-up.

\section{References}

1. De Hert, Dekker JM, Wood D, Kahl KG, Holt RIG, et al. (2009) Cardiovascular disease and diabetes in people with severe mental illness position statement from the European Psychiatric Association (EPA), supported by the European Association for the Study of Diabetes (EASD) and the European Society of Cardiology (ESC). Eur Psychiatry 24: 412-424.

2. Hex N, Bartlett C, Wright D, Taylor M, Varley D (2012) Estimating the curren and future costs of Type 1 and Type 2 diabetes in the UK, including direct health costs and indirect societal and productivity costs. Diabet Med 29: 855-862.

3. (2012) Diabetes in the UK 2012. Key statistics on diabetes. London, Diabetes UK

4. Department of Health (2001) National Diabetes Audit Mortality Analysis 20072008.

5. Surtees PG, Wainwright NWJ, Luben RN, Wareham NJ, Bingham SA, et al (2008) Depression and ischemic Heart Disease Mortality: Evidence from the EPIC-Norfolk United Kingdom Prospective Cohort Study. Am J Psychiatry 165 515-523.

6. ParkAL, McDaid D, Weiser P, Von Gottberg C, Becker T, et al. (2013) Examining the cost effectiveness of interventions to promote the physical health of people with mental health problems: a systematic review. BMC Public Health 13: 787.

7. Cabassa LJ, Ezell JM, Lewis-Fernández R (2010) Lifestyle interventions for adults with serious mental illness: a systematic literature review. Psychiatr Serv 61: 774-782

8. Papanastasiou E (2012) Interventions for the metabolic syndrome in schizophrenia: a review. Ther Adv Endocrinol Metab 3: 141-162.

9. National Institute for Health and Care Excellence (2014) Psychosis and Schizophrenia in Adults: The NICE Guideline on Treatment and Management in Adults. CG 178. London: National Institute for Health and Care Excellence.

10. Khazaal Y, Fresard E, Rabia S, Chatton A, Rothen S, et al. (2007) Cognitive behavioural therapy for weight gain associated with antipsychotic drugs Schizophr Res 91: 169-177.

11. Treeage Software Inc. (2014) Treeage Pro. Treeage Software Inc. Williamstown, MA.

12. McKibbin CL, Patterson TL, Norman G, Patrick K, Jin H, et al. (2006) Lifestyle intervention for older schizophrenia patients with diabetes mellitus: a randomized controlled trial. Schizophr Res 86: 36-44.

13. McKibbin CL, Golshan S, Griver K, Kitchen K, Wykes TL (2010) A healthy lifestyle intervention for middle-aged and older schizophrenia patients with diabetes mellitus: a 6-month follow-up analysis. Schizophr Res 121: 203-206.

14. NICE (2006) Obesity: Guidance on the Prevention, Identification, Assessment and Management of Overweight and Obesity in Adults and Children. NICE clinical guideline 43. London: NICE.

15. Cimo A, Stergiopoulos E, Cheng C, Bonato S, Dewa CS (2012) Effective lifestyle interventions to improve type II diabetes self-management for those with schizophrenia or schizoaffective disorder: a systematic review. BMC Psychiatry 12: 1-9.

16. Stratton IM, Adler AI, Neil HA, Matthews DR, Manley SE, et al. (2000) Association of glycaemia with macrovascular and microvascular complications of type 2 diabetes (UKPDS 35): prospective observational study. BMJ 321: 405 412.

17. PSSRU (2013) Unit Costs of Health and Social Care. University of Kent: Personal Social Services Research Unit.

18. Clarke PM, Gray AM, Briggs A, Stevens RJ, Matthews DR, et al. (2005) Cost- 
Citation: Park AL (2014) Exploring the Economic Implications of a Group-Based Lifestyle Intervention for Middle-Aged Adults with Chronic Schizophrenia and Co-Morbid Type 2 Diabetes. J Diabetes Metab 5: 366 doi:10.4172/2155-6156.1000366

utility analyses of intensive blood glucose and tight blood pressure control in type 2 diabetes (UKPDS 72). Diabetologia 48: 868-877.

19. Lenert LA, Sturley AP, Rapaport MH, Chavez S, Mohr PE, et al. (2004) Public preferences for health states with schizophrenia and a mapping function to estimate utilities from positive and negative symptom scale scores. Schizophr Res 71: 155-165.

20. Russell LB, Gold MR, Siegel JE, Daniels N, Weinstein MC (1996) The role of cost-effectiveness analysis in health and medicine. Panel on Cost-Effectiveness in Health and Medicine. JAMA 276: 1172-1177.

21. Box, GEP, Draper NR (1987) Empirical Model-Building and Response Surfaces: 424

22. Dinca-Panaitescu S1, Dinca-Panaitescu M, Bryant T, Daiski I, Pilkington B, et al (2011) Diabetes prevalence and income: Results of the Canadian Community Health Survey. Health Policy 99: 116-123.

23. Wilkinson R, Marmot M (2003) Social determinants of health: the solid facts. Denmark: World Health Organisation (WHO) regional office.
24. Dickerson FB, Goldberg RW, Brown $\mathrm{CH}$, Kreyenbuhl JA, Wohlheiter K, et al. (2005) Diabetes knowledge among persons with serious mental illness and type 2 diabetes. Psychosomatics 46: 418-424.

25. Lieberman JA, Stroup TS, McEvoy JP, Swartz MS, Rosenheck RA, et al. (2005) Effectiveness of antipsychotic drugs in patients with chronic schizophrenia. N Engl J Med 353: 1209-1223.

26. Thorgeirsson T, Kawachi I (2013) Behavioral economics: merging psychology and economics for lifestyle interventions. Am J Prev Med 44: 185-189.

27. Holt RI, Pendlebury J, Wildgust HJ, Bushe CJ (2010) Intentional weight loss in overweight and obese patients with severe mental illness: 8-year experience of a behavioral treatment program. J Clin Psychiatry 71: 800-805.

28. Molosankwe I, Patel A, José Gagliardino J, Knapp M, McDaid D (2012) Economic aspects of the association between diabetes and depression: a systematic review. J Affect Disord 142 Suppl: S42-55.

29. Tsapas A, Matthews DR (2008) N of 1 trials in diabetes: making individua therapeutic decisions. Diabetologia 51: $921-925$ 